\title{
EDITORIAL \\ Modified extended approach improves recovery following spinal accessory to suprascapular nerve transfer
}

\author{
Yuval Shapira, MD¹, and Rajiv Midha, MD, MSc ${ }^{2}$ \\ 'Department of Neurosurgery, Tel Aviv Medical Center, Tel Aviv, Israel; and 2Department of Clinical Neurosciences and \\ Hotchkiss Brain Institute, University of Calgary, Alberta, Canada
}

B RACHIAL plexus injuries are severe and often disabling injuries that occur in up to $5 \%$ of highenergy accidents. ${ }^{4}$ Selecting the appropriate treatment relies on accurate grading and classification of the injury, whether partial or complete and pre- versus postganglionic. ${ }^{6}$ One of the most common types of injury is complete brachial plexus injury with no function of the upper extremity (flail arm), which unfortunately portends a poor recovery. This injury pattern is typically associated with a high frequency of pre-ganglionic injury, with avulsion of multiple nerve roots. ${ }^{4}$ Surgical treatment of these patients usually includes nerve transfers, which are especially challenging because of the limited availability of donor nerves to support functional recovery. The typical extra-plexal donors are the spinal accessory nerve, phrenic nerve, contralateral C-7, or intercostal nerves. A close anatomical relationship between the donor and recipient nerve is important in order to avoid the need for an interposition nerve graft with additional coaptation sites and potential loss of axons and resulting in poorer outcomes. ${ }^{3}$

In complete brachial plexus injury, the most common technique for restoring shoulder stability, shoulder abduction, and external rotation is by neurotization of the spinal accessory nerve to the suprascapular nerve. Shoulder abduction is aided by supraspinatus muscle recovery and external rotation by infraspinatus muscle function, while reinnervation of rotator cuff muscles confers static and dynamic shoulder stability. In the study by Bertelli and Ghizoni ${ }^{1}$ published in this issue of the Journal of Neurosurgery: Spine, the authors present their extensive experience with and results of spinal accessory to suprascapular nerve transfers for shoulder stability, abduction, and external rotation in patients who have suffered complete brachial plexus injury.

This is a retrospective analysis of 110 cases involving patients operated on during 11 years by a single surgeon for shoulder stability and abduction reanimation by spinal accessory nerve to suprascapular nerve transfer together with restoration of elbow flexion by either spinal nerve root grafting, with or without phrenic nerve transfer or intercostal nerve transfer to the musculocutaneous nerve. During this period, the surgeon modified the surgical approach from a classic L-shaped incision in the early phase to an oblique/extended incision. The latter allows more distal exploration of the suprascapular nerve at the suprascapular notch and fossa and better exposure of the donor nerve (the spinal accessory nerve), thereby avoiding the use of an interposition nerve graft (used occasionally when utilizing the classic approach). Successful shoulder abduction was defined as at least $30^{\circ}$ during examination at least 24 months after the surgery. The authors identified a significantly higher success rate in range of shoulder abduction and also a much higher range of external rotation function in the group of patients who were treated utilizing the oblique/extended approach (those treated during the second part of the study period). Notably, the results for shoulder abduction are quite remarkable but with the caveat that the technique the authors use on video for measuring rotation includes both glenohumeral and scapulothoracic contributions, while others have attempted to measure "pure" glenohumeral abduction. ${ }^{2}$ Readers should also note that the results noted for external rotation are for the degrees of rotation away from complete internal rotation with the forearm held at baseline against the chest wall. Future series should compare results using these criteria; we agree with the authors that these criteria provide a reliable index of shoulder movement on a functional basis for activities of daily living.

The strength of this paper is the patient cohort, which represents the largest series to date of spinal accessory to supraspinatus nerve transfer in complete brachial plexus injury, comparing the classic L approach with the more 
modern, and now routinely utilized, ${ }^{5}$ oblique approach. The additional value of the paper is the concept of using an "extended" anterior approach to reach the supraspinatus nerve at the suprascapular notch area to find the nerve, which may be quite distal in in patients with very severe stretch injuries and avulsions. We too have employed this approach in several recent cases to find the nerve and to assess it for the rare "skip" lesion at the notch.

There are, however, some major limitations of this study that should be noted. First, this is an uncontrolled retrospective study of consecutive cases and includes surgeonevaluated outcomes, which may bias the results. Second, comparing a recent cohort to a more remote one adds a confounding issue related to the improved experience of the surgeon and more experienced supporting team, with better understanding and implementation of the rehabilitation process and physiotherapy methods (which are now receiving greater focus for restoring function following nerve transfer procedures). All of these factors may contribute to the improved outcome of the patients who were treated during the more recent period. Another possible confounding factor lies with the high percentage (more than 50\%) of patients who were excluded from the study due to insufficient follow-up. The authors do not provide any information regarding recovery for these patients. Theoretically it is possible that patients did not return to follow-up because of dissatisfaction with a poor outcome, or alternatively, as a result of excellent recovery, so the overall results may appear better or worse than they truly are.

Nevertheless, this is the largest series to date of spinal accessory to suprascapular nerve transfer in patients with complete brachial plexus injuries, and the authors provide valuable information and pearls for current and future management of such cases. The concept of an extended approach, which enables better anatomical exposure and evaluation of the donor spinal accessory nerve and recipient supraspinatus nerve, is a major advance. We congratulate the authors on their series and their results and encourage them to undertake future studies that assess this approach in a prospective fashion and also evaluate patients' quality of life and overall satisfaction.

http://thejns.org/doi/abs/10.3171/2015.9.SPINE15960

\section{References}

1. Bertelli JA, Ghizoni MF: Results of spinal accessory to suprascapular nerve transfer in 110 patients with complete palsy of the brachial plexus. J Neurosurg Spine [epub ahead of print February 12, 2016. DOI: 10.3171/2015.8.SPINE15434]

2. Malessy MJA, de Ruiter GCW, de Boer KS, Thomeer RTWM: Evaluation of suprascapular nerve neurotization after nerve graft or transfer in the treatment of brachial plexus traction lesions. J Neurosurg 101:377-389, 2004

3. Malessy MJA, Thomeer RTWM: Evaluation of intercostal to musculocutaneous nerve transfer in reconstructive brachial plexus surgery. J Neurosurg 88:266-271, 1998

4. Midha R: Epidemiology of brachial plexus injuries in a multitrauma population. Neurosurgery 40:1182-1189, 1997

5. Midha R, Alant JD de V: Nerve repair/nerve transfer strategies for adult brachial plexus palsies, in Chung KC, Yang LJS, McGillicuddy JE (eds): Practical Management of Pediatric and Adult Brachial Plexus Palsies. Edinburgh: Elsevier Saunders, 2012, pp 198-211
6. Yang LJS, Chang KWC, Chung KC: A systematic review of nerve transfer and nerve repair for the treatment of adult upper brachial plexus injury. Neurosurgery 71:417-429, 2012

\section{Disclosures}

The authors report no conflict of interest.

\section{Response}

\section{Jayme Augusto Bertelli, MD, $\mathrm{PhD},{ }^{1,2}$ and Marcos Flávio Ghizoni, MD, MSc ${ }^{1}$}

${ }^{1}$ Department of Neurosurgery, Southern University of Santa Catarina (Unisul), Tubarão; and 2Department of Orthopedic Surgery, Governador Celso Ramos Hospital, Florianópolis, Santa Catarina, Brazil

Of the 565 patients who underwent surgery for brachial plexus injuries during the period from 2002 to 2012 in our institution, $50 \%$ were found to have total palsy of the brachial plexus (Table 1). Such extended lesions mostly resulted from stretch injuries following motorcycle accidents. ${ }^{13}$ Unlike a clean cut, the stretch injury energy dissipates along the brachial plexus elements producing extended lesions. Hence, the possibility of extending the dissection up to a healthy distal nerve stump is crucial for success in an accessory to suprascapular nerve transfer. We always initiate dissection of the suprascapular nerve by the limited supraclavicular anterior approach. If we cannot find a healthy suprascapular nerve, we detach the trapezius from the clavicle to assess the suprascapular nerve retroclavicularly. If scar tissue prevents dissection, we expose the suprascapular nerve at the suprascapular fossa. Direct exposure of the suprascapular nerve at the suprascapular fossa saves time if the patient has clavicle fracture or dislocation or has had previous surgery. Dissection at this level is not easy, and venous bleeding should

TABLE 1. Type of palsy and root involvement after brachial plexus trauma in a series of 565 patients who underwent surgery at our institution from 2002 to $2012^{*}$

\begin{tabular}{cc}
\hline Type of Brachial Plexus Palsy & No. of Patients (\%) \\
\hline Supraclavicular injuries & $512(91)$ \\
\hline Total palsy & $257(50)$ \\
\hline Partial avulsion & $193(75)$ \\
\hline Total avulsion & $64(25)$ \\
\hline Upper type injuries & $240(47)$ \\
\hline C5-6 & $58(24)$ \\
\hline C5-7 & $48(20)$ \\
\hline C5-8 & $134(56)$ \\
\hline Lower type injuries & $15(3)$ \\
\hline C7-T1 & $10(67)$ \\
\hline C8-T1 & $5(33)$ \\
\hline Infraclavicular injuries & $53(9)$ \\
\hline partial avulsion, which means that a root was available for grafting in the \\
majority of our cases. In the upper type injuries note the higher prevalence of \\
C5-8 root injury.
\end{tabular}


be controlled without extensive bipolar use to avoid injury of the delicate motor branches. Veins at this level resemble the vertebral plexus, and control of bleeding may require elevation of the patient's head and the application of local hemostatic agents.

In 2004, we introduced the posterior approach for suprascapular nerve reconstruction in patients with brachial plexus lesions. ${ }^{3}$ Relative to the anterior approach, dissection of the suprascapular nerve was more difficult and occurred in a much deeper plane, roughly $10 \mathrm{~cm}$ from the skin surface. It should be stressed that the suprascapular notch is located underneath the clavicle and not close to the scapular spine. Using the posterior approach, there was no clear control of the suprascapular vessels. In addition, patients needed to be in a prone position at the beginning of the procedure but then be turned to a supine position for elbow flexion reconstruction, and the repositioning was time consuming. We therefore ultimately abandoned the posterior approach for routine exposure of the suprascapular nerve during brachial plexus surgeries. More recently, a few surgeons have proposed the posterior approach for accessory to suprascapular nerve transfers, $1,5,7,8$ its advantages being less denervation of the trapezius muscle and nerve coaptation closer to the muscles. However, the extended anterior approach permits coaptation at the same level, with greater comfort. The argument that the dorsal approach preserves trapezius strength also is questionable. While harvesting the spinal accessory nerve, provided that innervation of the upper trapezius nerve is preserved, postoperative trapezius strength is always maintained, as demonstrated in the 110 patients enrolled in our current study. Extended dissection of the spinal accessory nerve leads to a decreased number of fibers, from 1300 to $800,{ }^{14}$ for suprascapular nerve reconstruction. In total lesions of the brachial plexus, whether treated via the extended anterior or the posterior approach, the benefits of performing a distal accessory to suprascapular nerve coaptation closer to muscle targets need to be determined, given the decreasing number of regenerating fibers. Indeed, in electrophysiological studies involving 74 patients with brachial plexus injuries, Rui et al. ${ }^{10}$ observed no differences between the classic anterior and posterior approaches with respect to the time to infraspinatus reinnervation. Moreover, in 23 patients operated upon using the posterior approach, the mean range of abduction recovery was only $29^{\circ}$ an average of 32 months after spinal accessory to suprascapular nerve transfer. Data on abduction recovery with the classic anterior approach were not reported. The authors ${ }^{10}$ concluded that the posterior approach should be employed only when the classic anterior approach is not feasible.

In general, in order to avoid muscle stretching and glenohumeral subluxation, the use of an arm sling is recommended to patients with brachial plexus injury. ${ }^{11}$ However, the prolonged use of the arm sling leads to permanent elevation of the shoulder joint and clavicle, making dissection of the suprascapular nerve difficult (Fig. 1). In addition, the use of the arm sling leads to shoulder adduction and internal rotation contractures, which are very difficult to treat in these completely palsied limbs. We strongly discourage preoperative use of any arm sling and allow our

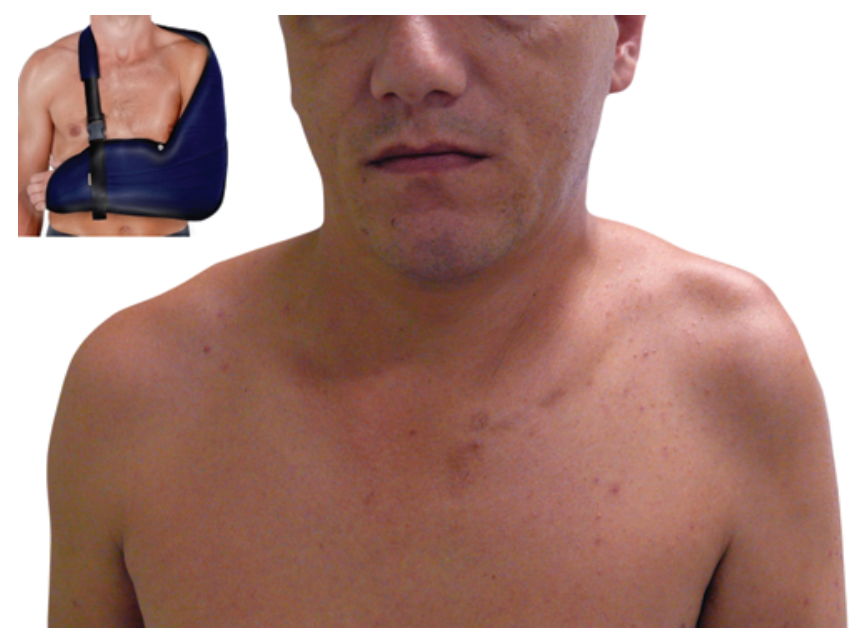

FIG. 1. Left shoulder elevation and adduction following the use of an arm sling in a patient with total palsy of the brachial plexus. The supraclavicular fossa is narrowed and the clavicle raised, which compromises suprascapular nerve dissection. Figure is available in color online only.

patients to use the arm sling for just a few days after surgery. It might be thought that by not using an arm sling to avoid supraspinatus stretching before re-innervation there might be some negative effect on recovery, but we have not found that to be the case. Moreover, it is possible that when patients do not use the arm sling, the supraspinatus muscle is stretched and that this stretching inhibits muscle atrophy. Experimentally it has been demonstrated that stretching of denervated muscle augments the production of actin and myosin filaments and the number of sarcomeres. $^{12}$

In our hands, among all the nerve transfers procedures available for treatment of total palsies of the brachial plexus, the accessory to suprascapular nerve transfer is the most reliable. In a large series of 110 patients we demonstrated an overall failure rate, meaning less than $30^{\circ}$ abduction, of only $9 \%$, with an average range of abduction recovery of approximately $60^{\circ}\left(\mathrm{SD} 25^{\circ}\right)$. Due to the complexity of shoulder motion, one-third of the motor fibers within the brachial plexus innervate shoulder muscles. ${ }^{6}$ Hence, it is not possible to obtain abduction recovery above horizontal by a single nerve transfer. In total palsies, abduction above horizontal is in fact not only impossible to achieve but would be useless if the hand remains paralyzed.

Complete palsies of the brachial plexus are devastating injuries and the possibilities for reconstruction are still very limited. Brachial plexus exploration and root grafting allow for immediate pain control in 50\% of patients and partial restoration of shoulder and elbow motion. ${ }^{2,4}$ These achievements, although modest, improve patients' quality of life. Unfortunately, objective measurements of the use of the treated limb in daily activities are lacking. ${ }^{9}$ One main reason for the lack of these clinimetric studies is that common instruments for assessment of upper-limb function, such as the DASH (Disabilities of the Arm Shoulder and Hand) questionnaire, poorly differentiate neurological recovery from adaptation in day-to-day tasks using the unaffected limb. ${ }^{9}$ 


\section{References}

1. Bahm J, Noaman H, Becker M: The dorsal approach to the suprascapular nerve in neuromuscular reanimation for obstetric brachial plexus lesions. Plast Reconstr Surg 115:240-244, 2005

2. Bertelli JA, Ghizoni MF: Pain after avulsion injuries and complete palsy of the brachial plexus: the possible role of nonavulsed roots in pain generation. Neurosurgery 62:11041114,2008

3. Bertelli JA, Ghizoni MF: Contralateral motor rootlets and ipsilateral nerve transfers in brachial plexus reconstruction. J Neurosurg 101:770-778, 2004

4. Bertelli JA, Ghizoni MF: Reconstruction of complete palsies of the adult brachial plexus by root grafting using long grafts and nerve transfers to target nerves. J Hand Surg Am 35:1640-1646, 2010

5. Bhandari PS, Deb P: Posterior approach for both spinal accessory nerve to suprascapular nerve and triceps branch to axillary nerve for upper plexus injuries. J Hand Surg Am 38:168-172, 2013

6. Bonnel F, Allieu Y, Sugata Y, Rabischong P: Bases anatomochirurgicales des neurotisations pour avulsions radiculaires du plexus brachial. Anat Clin 1:291-296, 1979

7. Colbert SH, Mackinnon S: Posterior approach for double nerve transfer for restoration of shoulder function in upper brachial plexus palsy. Hand (N Y) 1:71-77, 2006
8. Guan SB, Hou CL, Chen DS, Gu YD: Restoration of shoulder abduction by transfer of the spinal accessory nerve to suprascapular nerve through dorsal approach: a clinical study. Chin Med J 119:707-712, 2006

9. Hill BE, Williams G, Bialocerkowski AE: Clinimetric evaluation of questionnaires used to assess activity after traumatic brachial plexus injury in adults: a systematic review. Arch Phys Med Rehabil 92:2082-2089, 2011

10. Rui J, Zhao X, Zhu Y, Gu Y, Lao J: Posterior approach for accessory-suprascapular nerve transfer: an electrophysiological outcomes study. J Hand Surg Eur Vol 38:242-247, 2013

11. Saliba S, Saliba EN, Pugh KF, Chhabra A, Diduch D: Rehabilitation considerations of a brachial plexus injury with complete avulsion of C5 and C6 nerve roots in a college football player: a case study. Sports Health 1:370-375, 2009

12. Smania N, Berto G, La Marchina E, Melotti C, Midiri A, Roncari L, et al: Rehabilitation of brachial plexus injuries in adults and children. Eur J Phys Rehabil Med 48:483-506, 2012

13. Soldado F, Ghizoni MF, Bertelli JA: Mechanisms of trauma in supraclavicular stretch injuries of the brachial plexus. Ann Chir Main Memb Super [in press], 2015

14. Vathana T, Larsen M, de Ruiter GCW, Bishop AT, Spinner RJ, Shin AY: An anatomic study of the spinal accessory nerve: Extended harvest permits direct nerve transfer to distal plexus targets. Clin Anat 20:899-904, 2007 